Purdue University

Purdue e-Pubs

$1-1-2008$

\title{
Infrared Micro-Particle Image Velocimetry Measurements and Predictions of Flow Distribution in a Microchannel Heat Sink
}

Benjamin Jones

Purdue University, jones30@purdue.edu

Poh-Seng Lee

S V. Garimella

Purdue University, sureshg@purdue.edu

Follow this and additional works at: http://docs.lib.purdue.edu/coolingpubs

Jones, Benjamin; Lee, Poh-Seng; and Garimella, S V., "Infrared Micro-Particle Image Velocimetry Measurements and Predictions of Flow Distribution in a Microchannel Heat Sink" (2008). CTRC Research Publications. Paper 98.

http://dx.doi.org/10.1016/j.ijheatmasstransfer.2007.06.034

This document has been made available through Purdue e-Pubs, a service of the Purdue University Libraries. Please contact epubs@purdue.edu for additional information. 


\title{
Infrared Micro-Particle Image Velocimetry Measurements and Predictions of Flow Distribution in a Microchannel Heat Sink ${ }^{*}$
}

\author{
Benjamin J. Jones, Poh-Seng Lee, and Suresh V. Garimella ${ }^{\dagger}$ \\ School of Mechanical Engineering \\ Purdue University \\ West Lafayette, Indiana 47907-2088 USA
}

\begin{abstract}
The flow distribution in a silicon microchannel heat sink was studied using Infrared MicroParticle Image Velocimetry (IR $\mu$ PIV). The microchannel test piece consisted of seventy-six 110 $\mu \mathrm{m}$ wide $\times 371 \mu \mathrm{m}$ deep channels etched into a silicon substrate. Inlet and outlet manifolds, also etched into the substrate, were fed by $1.4 \mathrm{~mm}$ inner-diameter tubing ports. An image processing algorithm was developed that significantly improves the quality of IR $\mu$ PIV recordings in low signal-to-noise ratio environments. A general expression for the PIV measurement depth is presented, which is valid for PIV images that have undergone a threshold image processing operation. Experiments were performed at two different flow rates: $10 \mathrm{ml} / \mathrm{min}(\mathrm{Re}=10.2)$ and 100 $\mathrm{ml} / \mathrm{min}(\mathrm{Re}=102)$. Little flow maldistribution was observed at the lower flow rate. However, significant flow maldistribution was observed at $\operatorname{Re}=102$, with the channels near the centerline having an approximately $30 \%$ greater mass flux than the channels near the lateral edges of the heat sink. Numerical simulations carried out for flow in the microchannel heat sink agreed very well with the experimental measurements, validating the use of a computational approach for studying the effect of manifold design on flow distribution in microchannel heat sinks.
\end{abstract}

Keywords: Particle image velocimetry, microchannels, flow distribution, maldistribution, infrared, optical diagnostics

\footnotetext{
* Submitted for possible publication in International Journal of Heat and Mass Transfer, September 2006, and in revised form, June 2007

${ }^{\dagger}$ To whom correspondence should be addressed: sureshg@purdue.edu, 765-494-5621 (P), 765-494-0539 (F)
} 


\begin{tabular}{|c|c|c|c|}
\hline \multicolumn{2}{|c|}{ Nomenclature } & \multirow{2}{*}{$\begin{array}{l}\mathrm{u} \\
\mathrm{u}_{\mathrm{diff}}\end{array}$} & \multirow{2}{*}{$\begin{array}{l}\text { velocity } \\
\text { dimensionless diffraction variable }\end{array}$} \\
\hline $\mathrm{a}$ & aperture radius & & \\
\hline$a_{r}$ & aspect ratio & $\mathrm{W}_{\mathrm{c}}$ & channel width \\
\hline $\mathrm{d}_{\mathrm{e}}$ & effective particle diameter & & \\
\hline$d_{p}$ & particle diameter & Greek & \\
\hline$d_{s}$ & diffraction-limited spot size & $\alpha$ & illumination angle \\
\hline e & distance between pixels & $\alpha_{n}$ & eigenvalue \\
\hline $\mathrm{F}$ & optical fill factor & $\delta z_{\text {diff }}$ & contribution to $\delta z_{m}$ due to diffraction \\
\hline $\mathrm{f}$ & focal length & $\delta \mathrm{z}_{\mathrm{DOF}}$ & depth of field \\
\hline $\mathrm{H}_{\mathrm{c}}$ & channel height & $\delta z_{\text {geom }}$ & contribution to $\delta z_{m}$ due to geometry \\
\hline $\mathrm{I}_{\max }$ & maximum intensity & $\delta z_{\mathrm{m}}$ & PIV measurement depth \\
\hline $\mathrm{I}_{\mathrm{th}}$ & threshold intensity & $\theta$ & half-angle subtended by extreme rays \\
\hline M & magnification & & passing through microscope objective \\
\hline$\overline{\mathrm{m}}_{\mathrm{c}}^{\prime \prime}$ & area-averaged mass flux in a channel & $\lambda$ & wavelength \\
\hline NA & numerical aperture & $\lambda_{0}$ & wavelength in a vacuum \\
\hline $\mathrm{p}$ & pressure & $\mu$ & dynamic viscosity \\
\hline $\operatorname{Re}$ & Reynolds number based on hydraulic & $\rho$ & density of fluid \\
\hline & diameter of the channel & $\phi$ & threshold intensity ratio \\
\hline s & overall pixel dimension & & \\
\hline
\end{tabular}

\section{Introduction}

Microchannel heat sinks were first demonstrated by Tuckerman and Pease [1] as a highperformance cooling technique for microelectronics. The increase in cooling demands of highperformance microprocessors has generated increased interest in microchannel heats sinks over the past few years. Although the single-phase fluid flow and heat transfer characteristics in microchannels are now well understood [2-3], less attention has been devoted in the literature to flow distribution within the heat sink, which can adversely affect heat transfer performance. Numerical studies of flow maldistribution in minichannel and microchannel heat sinks have been performed [4-5]; well-characterized experimental results, however, have not been reported.

Accurate measurement of fluid flows in microchannel heat sinks requires diagnostic techniques with micron-scale spatial resolution. One such technique that has grown in popularity is microparticle image velocimetry ( $\mu \mathrm{PIV})$. The technique was first demonstrated by Santiago et al. [6]. 
Measurements of the velocity field around a $30 \mu \mathrm{m}$ elliptical cylinder were made in Hele-Shaw flow with a bulk velocity of approximately $50 \mu \mathrm{m} / \mathrm{s}$. A spatial resolution of $6.9 \times 6.9 \times 1.5 \mu \mathrm{m}$ was achieved. The technique was further demonstrated in a microchannel by Meinhart et al. [7], where a spatial resolution of $13.6 \times 0.9 \times 1.8 \mu \mathrm{m}$ was achieved. The results agreed to within $2 \%$ of the analytical solution for laminar flow in a rectangular microchannel.

The use of $\mu$ PIV has been limited to applications in which optical access to the fluid flow is available at visible wavelengths. In some applications such as stacked microchannel heat sinks, optical access to the fluid flow in the visible spectrum is unavailable. Since silicon is quite transparent to wavelengths between 1100 and $2500 \mathrm{~nm}$, a diagnostic technique in the infrared range would be advantageous for measuring subsurface flow fields in silicon microdevices. Han et al. [8] first demonstrated an infrared $\mu \mathrm{PIV}$ (IR $\mu \mathrm{PIV}$ ) technique by measuring the velocity field in a micronozzle with a $300 \mu \mathrm{m}$ depth and $40 \mu \mathrm{m}$ throat width. The solid motion of a microrotor was also measured with this infrared diagnostic system. The IR $\mu$ PIV technique was further discussed by Liu et al. [9], who obtained quantitative measurements in microtubes at various flow rates. The measurements were shown to agree well with laminar flow theory.

Although the IR $\mu$ PIV technique has shown great promise, implementation of IR $\mu$ PIV can be difficult due to a low signal-to-noise ratio. The two primary objectives of the present work are: 1) to present further developments to the IR $\mu$ PIV technique, and 2) to study flow maldistribution in a silicon microchannel heat sink using this technique. The flow maldistribution is further explored using computational fluid dynamics modeling. 


\section{Experimental Setup}

\subsection{IR $\mu$ PIV System}

The IR $\mu$ PIV system layout is shown in Figure 1. Infrared light from a wavelength-tunable, twin ND:YAG laser system is delivered to the micro-device through a fiber optic cable. A 20X magnification, 0.4 NA microscope objective, corrected for light in the $480 \mathrm{~nm}$ to $1800 \mathrm{~nm}$ range, collects the light scattered by the seed particles. A near-infrared (NIR) camera captures the particle images. The NIR camera employs a $320 \times 256$ pixel, indium-gallium arsenide focal plane array that is highly sensitive to wavelengths between 900 and $1700 \mathrm{~nm}$. The pixel size is $30 \times 30 \mu \mathrm{m}$ with an optical fill factor greater than $90 \%$. As discussed in Liu et al. [9], the choice of tracer particles is a compromise between flow traceability and particle visibility. A particle diameter of $2.0 \mu \mathrm{m}$ was found to be adequate for the present study. The visibility of the particles is also greatly affected by the incident angle of the fiber optic delivery, $\alpha$. The optimum incident angle is dependent on the light scattering characteristics of the tracer particles, which depend on the refractive index of the fluid and particle, the particle diameter, and illumination wavelength. Light scattering from the surfaces of the heat sink may also influence the optimum angle if the surfaces are not optically smooth. For the present work, $\alpha \approx 30^{\circ}$ was found to yield acceptable results.

The twin laser system generates two collinear beams $4.9 \mathrm{~ns}$ in duration at $1064 \mathrm{~nm}$. The laser beam is frequency-doubled to $532 \mathrm{~nm}$. The pumping beam $(532 \mathrm{~nm})$ is directed through an optical parametric oscillator (OPO), a non-linear optical crystalline device that converts the pumping beam into signal and idler beams. The wavelength of these beams can be tuned from $680 \mathrm{~nm}$ to $2400 \mathrm{~nm}$ by adjusting the angle of the OPO crystal. The optical performance of the PIV system can therefore be optimized by adjusting the output wavelength from the laser system. For the present work, satisfactory results were obtained using a laser wavelength of $1350 \mathrm{~nm}$.

\subsection{Experimental Setup}

Experiments were performed on the microchannel test piece shown in Figure 2. The test piece consists of 76 deep reactive ion etched (DRIE) channels in a silicon substrate with inlet and outlet manifold sections. As seen in Figure 2a, a glass cover plate is anodically bonded to the silicon substrate. Although the glass is transparent to visible light, the particles are illuminated through the silicon substrate (see Figure 1) in a configuration that would not be possible using standard $\mu$ PIV. If a silicon cover were used instead of glass, more laser power would be required due to the lower transmissivity of silicon than glass (about 50\% and 90\%, respectively). However the signal-to- 
noise ratio is not expected to be adversely affected as long as there is sufficient laser power, due to the small extinction coefficient of silicon at the wavelengths of interest $\left(<10^{-6}\right)[10]$. Holes were drilled through the cover glass, and stainless steel tubing ports with a $1.4 \mathrm{~mm}$ inner diameter were bonded to the glass using epoxy. PVC tubing was inserted over the tubing ports and secured using steel wire.

The dimensions of the heat sink are shown in Figure 2b. The manifold is $2 \mathrm{~mm}$ long with a recessed area for the tubing ports. The channels are $9 \mathrm{~mm}$ long. The naming convention for the channels and coordinate system is also presented in Figure $2 \mathrm{~b}$. IR $\mu$ PIV measurements were performed on the channels in the $-\mathrm{y}$ direction, with channel 1 being at the centerline and channel 38 being adjacent to the lateral edge. Channels in the $+y$ direction are referred to as -1 through -38 .

The microchannel test piece was sectioned and observed using an optical microscope to determine the channel width. Although the nominal channel width was $100 \mu \mathrm{m}$ with a $32 \mu \mathrm{m}$ thick fin, due to undercutting during the etch process, the actual channel width is $110 \mu \mathrm{m}$ over most of the channel height with the fin being $22 \mu \mathrm{m}$ thick. The average channel depth is $371 \mu \mathrm{m}$, as measured with an optical microscope at the mid-length of the channel $(x=4.5 \mathrm{~mm})$. As seen in Figure 3, there is some variation in channel depth from channel to channel, with the deeper channels being near the outer edges of the heat sink. The channel depth is within $\pm 4 \%$ of the average except near the $-\mathrm{y}$ edge, where the channel depth is $11 \%$ greater than the average channel depth. The effects of the variation in the channel depth on the results will be discussed in section 5. Measurements of the manifolds revealed that they are slightly deeper than the channels. The depth is fairly uniform over most of the manifold area, with an average depth of approximately $421 \mu \mathrm{m}$.

Experiments were performed at two flow rates: $10 \mathrm{ml} / \mathrm{min}$ and $100 \mathrm{ml} / \mathrm{min}$, corresponding to average channel Reynolds numbers of 10.2 and 102, respectively, based on a hydraulic diameter of $170 \mu \mathrm{m}$. The lower flow rate $(\mathrm{Re}=10.2)$ was provided by a syringe pump. For the higher flow rate experiments $(\operatorname{Re}=102)$, a pressure-driven flow loop supplied the fluid/particle mixture to the heat sink (see Figure 4). A pressure vessel containing the fluid was pressurized using compressed air, forcing the particle mixture through the flow loop. A high-precision pressure regulator was used to control the pressure in the vessel. A throttle valve allowed fine tuning of the flow rate. The differential pressure across the microchannel test piece was monitored using a data acquisition system (DAQ) to ensure that a uniform flow rate was maintained during the course of the experiment. 
The particle mixture was collected in a beaker. The mass of the beaker was measured before and after each experiment. Accurate timing was achieved by deducing when the valve was opened and closed based on the pressure data collected from the DAQ. The accuracy of the calculated flow rate is estimated to be $\pm 0.2 \%$. The flow rates for all of the experiments were within $\pm 2 \%$ of the set point. Each PIV measurement was corrected according to the measured flow rate. The accuracy of the syringe pump used for the lower flow rate was estimated to be within $\pm 0.3 \%$ of the set point.

\section{Imaging and Analysis}

\subsection{Image Processing}

In $\mu \mathrm{PIV}$, inelastic scattering techniques such as epi-fluorescence are commonly used to increase the signal-to-noise ratio. However, to the authors' knowledge, epi-fluorescent dyes in the necessary wavelength range for IR $\mu$ PIV are currently unavailable. Since elastic scattering must be used, IR $\mu \mathrm{PIV}$ typically requires more advanced image-processing techniques than $\mu$ PIV. An imageprocessing algorithm that significantly improves the image quality prior to PIV analysis was developed as detailed in this section.

Due to a fixed dead time of $762 \mathrm{~ms}$ between frames on the NIR camera, auto-correlation was required for the flow rates used in this work [9]. The original double-exposed PIV image taken in one of the microchannels is shown in Figure 5a. The fins are at the top and bottom of the image and the channel is in the center. A white haze is observed adjacent to the fins. This is an out-of-focus optical effect caused by light scattering at the interface between the silicon fin and glass cover. It is obvious that the contrast between the background and the particles is quite poor, with the particles being hardly visible above the background.

It is desirable to remove the background from the images to reduce its effect on the correlation function. The background can be removed by averaging all of the images in the recording and subtracting the averaged image from the original recording. The average image is shown in Figure $5 \mathrm{~b}$ and the result of the background removal is shown in Figure 5c. The resulting image after background removal is still quite noisy. A $5 \times 5$ unsharp mask (see Figure $5 \mathrm{~d}$ ) is used to reduce the effects of the low-frequency background noise. The unsharp mask operation is described in more detail by Lim [11].

The intensity of most of the particles is now sufficiently higher than the background intensity so that the particle images can be separated using a threshold where

$$
I_{t h}=\phi I_{\max }
$$


All pixel values below the threshold intensity, $\mathrm{I}_{\mathrm{th}}$, are set to 0 . The result of the threshold operation is shown in Figure 5e. The threshold intensity ratio, $\phi$, is chosen by trial and error until a reasonable compromise between noise removal and preservation of most of the particle image information is achieved. A threshold intensity ratio of 0.3 was found to be suitable for most of the image recordings in the present work.

Most of the background noise is removed as a result of these operations. However, there is a large variation in the intensity of the particle images, which can cause biasing of the correlation function. A local contrast enhancement technique is used to improve the uniformity of the particle image intensity. This technique first identifies the locations of the local intensity maxima in the image by finding pixel locations where all of the surrounding pixels are at a lower intensity. The contrast is enhanced in a region around each local maximum. The result of the local contrast enhancement is shown in Figure 5f.

The quality of the PIV record is thus markedly improved. However, some of the particles are removed due to the threshold value chosen. This results in some unpaired particles, where one of the particles of a double-exposed particle pair is removed. A rectangular search region is defined around each particle. If a neighbouring particle is not found within the search region, the particle is removed. The result of this operation is shown in Figure 5g.

The unpaired particle removal algorithm works best for low particle concentrations since this reduces the likelihood that a neighbouring particle pair will be located within the search region around an unpaired particle. As discussed in Meinhart et al. [12], low particle concentrations also have the added benefit of reducing the signal-to-noise ratio, especially in deep channels. A $0.01 \%$ particle concentration was found to yield suitable results.

\subsection{Depth of Field and Measurement Depth}

The depth of field is the out-of-plane distance that an object can move before it becomes unfocused. Inoué and Oldenbourg [13] derived an expression for the depth of field by considering both the diffraction and geometric effects:

$$
\delta \mathrm{z}_{\mathrm{DOF}}=\frac{\mathrm{n} \lambda_{0}}{\mathrm{NA}^{2}}+\frac{\mathrm{ne}}{\mathrm{NAM}}
$$

For a $\mathrm{CCD}$, e is the distance between pixels. However, it is often more convenient to express equation (2) in terms of the overall pixel dimension, s, and the optical fill factor, F, for CCD cameras. The optical fill factor is the ratio of the active pixel area to the overall pixel area. If the overall and active pixel areas are assumed to be squares, the following expression is obtained: 


$$
\delta \mathrm{z}_{\mathrm{DOF}}=\frac{\mathrm{n} \lambda_{0}}{\mathrm{NA}^{2}}+\frac{\mathrm{ns}(1-\sqrt{\mathrm{F}})}{\mathrm{NA} \mathrm{M}}
$$

In the current work, $\mathrm{n}=1$ for $\operatorname{air}, \lambda_{0}=1350 \mathrm{~nm}, \mathrm{NA}=0.4, \mathrm{M}=20, \mathrm{~s}=30 \mu \mathrm{m}$, and $\mathrm{F}=0.9$, resulting in a depth of field of $\delta z_{\mathrm{DOF}}=8.6 \mu \mathrm{m}$.

The measurement depth of a volume-illuminated PIV system is twice the distance from the center of the object plane to a location where particles no longer contribute significantly to the correlation function. The measurement depth effectively defines the out-of-plane spatial resolution of the PIV system. By considering the effects of diffraction, geometric optics, and finite particle size, the following expression for the measurement depth was derived by Meinhart et al. [12]:

$$
\delta \mathrm{z}_{\mathrm{m}}=\frac{3 \mathrm{n} \lambda_{0}}{\mathrm{NA}^{2}}+\frac{2.16 \mathrm{~d}_{\mathrm{p}}}{\tan \theta}+\mathrm{d}_{\mathrm{p}}
$$

Equation (4) assumes that particles no longer contribute significantly to the correlation function if the maximum intensity of the particle is one-tenth of the maximum in-plane particle intensity. Since the threshold intensity used in the image-processing algorithms is greater than the arbitrarily chosen intensity cut-off ratio, equation (4) is not strictly valid for the present work. Equation (4) over predicts the measurement depth by a sizeable margin. Therefore, it is advantageous to modify equation (4) such that the threshold intensity ratio, $\phi$, is an independent variable rather than an arbitrarily chosen value.

As discussed by Meinhart et al. [12], the contribution of diffraction to the measurement depth can be evaluated by considering the three-dimensional diffraction pattern from imaging a point source through a circular aperture. Along the optical axis, the diffraction pattern reduces to

$$
\frac{\mathrm{I}\left(\mathrm{u}_{\mathrm{diff}}\right)}{\mathrm{I}_{\max }}=\phi=\left(\frac{\sin \left(\mathrm{u}_{\mathrm{diff}} / 4\right)}{\mathrm{u}_{\mathrm{diff}} / 4}\right)^{2}
$$

where

$$
u_{\text {diff }}=2 \pi \frac{z}{\lambda}\left(\frac{a}{f}\right)^{2}
$$

Equation (5) cannot be explicitly solved for $\mathrm{u}_{\text {diff. }}$ In Meinhart et al. [12], $\phi$ was chosen to be 0.1 yielding $\mathrm{u}_{\mathrm{diff}} \approx 3 \pi$. However, a more general solution to equation (5) can be obtained using the first two terms of the Taylor series expansion of the sine function:

$$
\frac{\sin \left(\mathrm{u}_{\text {diff }} / 4\right)}{\mathrm{u}_{\mathrm{diff}} / 4} \approx 1-\frac{1}{6}\left(\frac{\mathrm{u}_{\mathrm{diff}}}{4}\right)^{2}=\sqrt{\phi}
$$


Substituting Eq. (6) into Eq. (7) and using the definition $\delta z_{\text {diff }}=2 z, f / a \approx n / N A$, and $\lambda_{0}=\lambda / n$ yields

$$
\delta \mathrm{z}_{\text {diff }}=3.12 \sqrt{1-\sqrt{\phi}} \frac{\mathrm{n} \lambda_{0}}{\mathrm{NA}^{2}}
$$

Meinhart et al. [12] showed that the contribution due to geometric optics can be written for an arbitrary value of $\phi$ as

$$
\delta z_{\text {geom }}=\frac{1-\sqrt{\phi}}{\sqrt{\phi}} \frac{d_{p}}{\tan \theta}, \text { for } d_{p}>\frac{e}{M}
$$

Equation (9) is valid for particle diameters greater than the distance between pixels projected back onto the object plane, which is common for most $\mu$ PIV applications.

The measurement depth is the summation of the contributions due to diffraction, geometric optics, and finite particle size. Using $\tan \theta \approx \mathrm{NA} / \mathrm{n}$ and equations (8) and (9) gives

$$
\delta \mathrm{z}_{\mathrm{m}}=3.12 \sqrt{1-\sqrt{\phi}} \frac{\mathrm{n} \lambda_{0}}{\mathrm{NA}^{2}}+\frac{1-\sqrt{\phi}}{\sqrt{\phi}} \frac{\mathrm{nd}_{\mathrm{p}}}{\mathrm{NA}}+\mathrm{d}_{\mathrm{p}}
$$

For $\phi=0.3$ and $\mathrm{d}_{\mathrm{p}}=2.0 \mu \mathrm{m}$, equation (10) results in a measurement depth, $\delta \mathrm{z}_{\mathrm{m}}$, of $23.8 \mu \mathrm{m}$. This compares to a measurement depth of $38.1 \mu \mathrm{m}$ given by equation (4). The measurement depth in the current work is considerably larger than what is typically achieved with conventional $\mu$ PIV systems. This is due to several factors: (i) the increased effects of diffraction due to longer wavelengths, (ii) the larger particle diameters necessitated by the need to increase the particle visibility, and (iii) the relatively low numerical aperture of the objective lens used. However, air-immersion lenses corrected for infrared wavelengths with numerical apertures up to 0.8 are available. Such a lens would yield a measurement depth of $7.8 \mu \mathrm{m}$ if all other parameters in Eq. (10) remained the same.

\subsection{Measurement Uncertainty}

According to Prasad et al. [14], the measurement error is minimized when the particle image diameter projected onto the CCD is approximately twice the pixel diameter. If such a condition is achieved, the position of the correlation peak can be determined to within one-tenth of the particle image diameter. The effective diameter of the particle projected onto the $\mathrm{CD}, \mathrm{d}_{\mathrm{e}}$, is the convolution of the diffraction with the geometric image of the particle:

$$
\mathrm{d}_{\mathrm{e}}=\sqrt{\mathrm{M}^{2} \mathrm{~d}_{\mathrm{p}}^{2}+\mathrm{d}_{\mathrm{s}}^{2}}
$$

where $d_{s}$ is the diffraction-limited spot size. Meinhart and Wereley [15] recommended the following relation for infinity-corrected optics commonly used in $\mu$ PIV applications: 


$$
\mathrm{d}_{\mathrm{s}}=1.22 \mathrm{M} \lambda \sqrt{(\mathrm{n} / \mathrm{NA})^{2}-1}
$$

For the present work, $\mathrm{d}_{\mathrm{e}} \approx 85 \mu \mathrm{m}$, resulting in $\mathrm{d}_{\mathrm{e}} / \mathrm{d}_{\mathrm{pix}} \approx 3$ for a $30 \mu \mathrm{m}$ pixel, which is close to the recommended value. Following Prasad et al. [14], this would result in a positional uncertainty of $0.43 \mu \mathrm{m}$ projected back into the object plane. For a maximum particle displacement of $15 \mu \mathrm{m}$ in the present work, this results in a minimum velocity uncertainty of approximately $3 \%$. However, more advanced PIV algorithms, such as continuous window shift and central difference interrogation which are implemented in the current work, have been developed since Prasad et al.'s recommendations, and this method of uncertainty estimation is questionable, although commonly employed in the literature.

\section{Numerical Analysis}

Recent studies $[3,16]$ have shown that computational fluid dynamics (CFD) analyses, when coupled with carefully matched entrance and boundary conditions, can be employed with confidence for predicting convective heat transfer behavior in microchannels. In this work, a similar approach was adopted except that the entire microchannel heat sink was included in the model, which consisted of inlet and outlet manifolds and the seventy-six parallel microchannels.

The computational domain is shown in Figure 6. The geometry of the heat sink is identical to that of the heat sink used in the experiments (as shown in Figure 2) albeit with some simplifications to facilitate easier mesh generation. Symmetry boundaries were used to reduce the extent of the computational model to the symmetric half-domain. There is no convective flux across the symmetry plane, so that the normal velocity component at the symmetry plane was zero. There is also no diffusion flux across the symmetry plane, rendering the normal gradients of all flow variables to be zero at the symmetry plane.

The channels were modeled with a uniform depth of $371 \mu \mathrm{m}$ (corresponding to the average measured channel depth discussed in Section 2.2) and width of $110 \mu \mathrm{m}$. The channels are separated by $22 \mu \mathrm{m}$ fins. The manifolds were modeled with a uniform depth of $421 \mu \mathrm{m}$. The inlet and outlet ports were modeled as cylindrical surfaces cut through the manifold regions. A uniform pressure was specified on these cylindrical surfaces and the pressure differential was adjusted to achieve the desired mass flow rate.

To simplify the analysis, the following assumptions were made in modeling the fluid flow in the microchannel test piece: steady state, incompressible fluid, laminar flow, and constant fluid properties. The continuity equation and the Navier-Stokes equations in their steady, incompressible 
form, along with the associated boundary conditions, were solved using the general-purpose finitevolume-based computational fluid dynamics (CFD) software package, FLUENT [17]. The SIMPLE algorithm was employed for pressure-velocity coupling. The momentum equations were solved with second-order up-wind differencing while the pressure terms were discretized using the standard scheme. The simulations were considered to have converged when the mass flow rate through each channel reached a steady value.

The domain was meshed with hexahedral elements, and a total of 3,497,468 cells were generated. Each channel had a $10 \times 15 \times 100$ grid $(\mathrm{x} \times \mathrm{y} \times \mathrm{z})$ with a two-sided successive ratio of 1.05 along the flow (x-) direction. Meshing at the junction of the manifold and channels is illustrated in Figure 6b. To establish the grid independence of the results, a finer mesh $(5,934,765$ cells) was also studied. For the high flow rate case $(\operatorname{Re}=102)$, both the maximum change in the flow rate in any given channel and the pressure required to achieve the targeted mass flow rate deviated less than $1 \%$ with respect to the coarser mesh $(3,497,468$ cells $)$. Therefore, the coarser mesh was considered sufficiently refined for the purposes of this study.

\section{Results and Discussion}

\subsection{Experimental Results}

The PIV analysis was carried out using a software program developed by the authors. The PIV code implemented central difference interrogation [18] and continuous window shifting [19]. The 300 images captured in each PIV recording were ensemble-averaged [20]. A $32 \times 8$ pixel interrogation window was used in the PIV analysis. The $1.481 \mu \mathrm{m} /$ pixel object-to-image dimensional ratio results in a spanwise spatial resolution of $11.8 \mu \mathrm{m}$.

Velocity profiles at $18 \mathrm{x}$-locations were evaluated in the fully developed region of the channel (between $\mathrm{x}=4.29 \mathrm{~mm}$ and $\mathrm{x}=4.69 \mathrm{~mm}$ ). The mean of the 18 velocity profiles in channel 35 at a flow rate of $\mathrm{Re}=102$ is shown in Figure 7. The error bars indicate the RMS error of the velocity profiles from the mean. The RMS error provides an estimate of the random error in the measurement. Also plotted is the analytical solution for the laminar velocity profile in a rectangular tube at mid-depth:

$$
\mathrm{u}=\mathrm{u} *(\mathrm{y}, \mathrm{z}=0) \frac{\mathrm{u}_{\max }}{\mathrm{u} *(\mathrm{y}=0, \mathrm{z}=0)}
$$

where $\mathrm{u}_{\max }$ is the maximum velocity determined experimentally from the PIV measurements and $\mathrm{u}^{*}$ is the non-dimensional velocity profile presented by Whitham [21]: 


$$
u^{*}=-\frac{\mathrm{u}}{\frac{\mathrm{W}_{\mathrm{c}}^{2}}{\mu} \frac{\mathrm{dp}}{\mathrm{dx}}}=\frac{1}{2}\left[\mathrm{a}_{\mathrm{r}}^{2}-\left(\frac{\mathrm{z}}{\mathrm{W}_{\mathrm{c}} / 2}\right)^{2}\right]+\frac{2}{\mathrm{a}_{\mathrm{r}}} \sum_{\mathrm{n}=1}^{\infty} \frac{(-1)^{\mathrm{n}}}{\alpha_{\mathrm{n}}^{3}} \cos \left(\alpha_{\mathrm{n}} \frac{\mathrm{z}}{\mathrm{W}_{\mathrm{c}} / 2}\right)^{\cosh \left(\alpha_{\mathrm{n}} \frac{\mathrm{y}}{\mathrm{W}_{\mathrm{c}} / 2}\right)} \frac{\cosh \left(\alpha_{\mathrm{n}}\right)}{\cos }
$$

where $\alpha_{\mathrm{n}}=(2 \mathrm{n}-1) \pi / 2 \mathrm{a}_{\mathrm{r}}, \mathrm{a}_{\mathrm{r}}=\mathrm{H}_{\mathrm{c}} / \mathrm{W}_{\mathrm{c}}$ is the aspect ratio, and $\mathrm{dp} / \mathrm{dx}$ is the pressure drop.

The match between the analytical solution and the experimental velocity profile is quite good for $|\mathrm{y}|< \pm \mathrm{W}_{\mathrm{c}} / 4$. The RMS error is less than $1.2 \%$ over this region. However, there is significant biasing of the velocities near the edges of the channel. The haze near the edges of the channel (see Figure 5a) results in a lower signal-to-noise ratio and reduces the number of particles successfully detected by the image processing algorithm. This causes a non-uniform particle distribution within the interrogation windows near the edge of the channel, which produces velocity measurements that are biased towards the more numerous, faster moving particles toward the center of the channel.

The flow distribution is shown in Figure 8. Velocity profiles in various channels across the heat sink are shown in Figure $8 \mathrm{a}$ for $\mathrm{Re}=10.2$ and Figure $8 \mathrm{~b}$ for $\mathrm{Re}=102$. Also plotted in Figure $8 \mathrm{a}$ and Figure $8 \mathrm{~b}$ are the mean theoretical velocity profiles evaluated from the known total flow rate through the heat sink and channel dimensions. Very little flow maldistribution is observed at the lower flow rate $(\operatorname{Re}=10.2)$, at which all of the measured velocity profiles fall very close to the mean velocity profile. At the higher flow rate $(\mathrm{Re}=102)$, much higher velocities are observed in channels near the centerline than towards the lateral edge.

Every PIV measurement was performed three times to ensure repeatability. The maximum difference in the area-averaged mass flux, $\rho \overline{\mathrm{u}}_{\mathrm{c}}$, calculated from the different data runs was less than $1.2 \%$ for $\operatorname{Re}=10.2$ and less than $2.5 \%$ for $\operatorname{Re}=102$. Figure $8 \mathrm{c}$ shows the ratio of the area-averaged mass flux determined from the PIV measurements to the average mass flux determined from the known total flow rate and average channel dimensions $\left(\rho \overline{\mathrm{u}}_{\mathrm{c}} / \overline{\mathrm{m}}_{\mathrm{c}}^{\prime \prime}\right)$. Measurements were also made (not shown) on the other side of the heat sink (channel -1 through -38) to check for symmetry at the lower flow rate $(\mathrm{Re}=10.2)$. The maximum deviation in mass flux ratio between a channel and its symmetric counterpart was only $2.2 \%$ despite channel -38 having an $8 \%$ greater depth than channel 38. This indicates that increased channel depth on the $+y$ side of the heat sink (channels -1 through -38) has a measurable but small impact on the flow distribution results (i.e., the mass flux ratio is approximately symmetric about the centerline of the heat sink).

The maximum mass flux ratio is approximately $2.4 \%$ greater than the minimum for $\operatorname{Re}=10.2$ and $29.9 \%$ greater for $\operatorname{Re}=102$. For $\operatorname{Re}=102$, channels 1 through 15 carry approximately $55 \%$ of 
the mass flow rate despite representing only $39 \%$ of the channels. It may be noted that the minimum measured mass flux ratio for $\mathrm{Re}=102$ occurs in channel 30, with channel 38 having approximately a $1.7 \%$ greater mass flux ratio than channel 30 . As shown in Figure 3, channel 38 has roughly a $4 \%$ greater channel depth than channel 30 , which may partially explain this difference in the mass flux ratio for these two channels.

\subsection{Numerical Results}

Figure 9 shows a comparison of the experimental mass flux ratio results with the numerically predicted values. The numerical results closely match the experimental measurements with a RMS deviation of $1.4 \%$ for $\operatorname{Re}=10.2$ and $2.4 \%$ for $\operatorname{Re}=102$. The differences between the experimental and numerical results can be attributed to simplifications in the numerical model and imperfections in the fabrication of the microchannel test piece. In particular, as discussed in section 2.2, the channels near the lateral edge of the heat sink are deeper than channels near the centerline. This results in more flow being diverted to the channels near the lateral edge. Since a uniform channel depth was specified in the numerical model, the numerical results would be expected to under predict the mass flux ratio for channels near the lateral edge and over predict the mass flux ratio for channels near the centerline, as is seen in Figure 9.

Furthermore, since channels in the $+y$ direction are typically deeper than those for which the experimental results are presented ( $-\mathrm{y}$ direction), more flow is diverted to channels on the $+\mathrm{y}$ side of the heat sink. Since the mass flux ratio is defined as the measured mass flux through each channel divided by the average mass flux through all of the channels in the entire heat sink, channels in the $-\mathrm{y}$ direction are expected to have a lower mass flux ratio than their symmetric counterpart. This may explain why the experimental results for the mass flux ratio typically fall below the numerical predictions, most notably for the higher Reynolds number case.

The manifold design (Figure 6) appears to cause a flow restriction at the lateral edges resulting in reduced flow rates in the channels in this region. To examine the influence of the manifold shape, a heat sink with a rectangular manifold design as shown in Figure 10a was also investigated numerically. The dimensions of the heat sink and associated boundary conditions are maintained the same as in Figure 6a, with the only difference being in the manifold shape.

The predicted mass flux ratios at $\mathrm{Re}=102$ with the original and rectangular manifold designs are compared in Figure 10b. The rectangular manifold clearly provides a much more uniform flow distribution through the heat sink than the original design. The numerical results predict a much reduced discrepancy of $4.7 \%$ between the maximum and minimum mass flux through the channels 
with the rectangular manifold, compared to a value of $33.5 \%$ with the original manifold design. It is also interesting to note that the static pressure drop across the heat sink (measured from the inlet port boundary condition to the outlet port boundary condition) for the original manifold is $32.7 \%$ greater than with the rectangular manifold ( $8.23 \mathrm{kPa}$ vs. $6.20 \mathrm{kPa}$, respectively). The pressure drop across the channels (measured between the channel inlet and exit) for the original manifold design ranges from $5.03 \mathrm{kPa}$ to $6.50 \mathrm{kPa}$ for channels 38 and 1, respectively, while the channel pressure drop for the rectangular manifold design varies over a much smaller range of $5.57 \mathrm{kPa}$ for channel 38 to $5.83 \mathrm{kPa}$ for channel 1 . The rectangular manifold design is clearly able to supply more uniform pressure at the channel inlets and exits.

The reasons for the superior flow performance of the rectangular manifold design are more clearly brought out in Figure 11. Figure 11a shows the numerical results for the original manifold while Figure 11b shows the results for the rectangular manifold; the upper portion of each figure shows the streamlines while the lower portion shows velocity magnitude contours. All results are shown at mid-height with respect to the channels. The streamlines in the rectangular manifold indicate that the flow distributes through the manifold easily and supplies all of the channels with a nearly uniform flow rate. The velocities at all locations in the manifold remain low, with lower associated pressure drops. For the original manifold design, on the other hand, a recirculation region is identified in the inlet manifold. This constricts the available space into which the fluid is distributed, resulting in higher localized velocities and larger pressure drops within the manifold. The fluid feeding the channels near the centerline has a more direct path through the manifold, so that the central channels receive a larger portion of the flow than channels near the lateral edge.

\section{Conclusions}

The flow distribution in a 76-channel heat sink with average channel dimensions of $110 \mu \mathrm{m} \times$ $371 \mu \mathrm{m}$ was studied using IR $\mu$ PIV. An image-processing algorithm was developed that significantly improves the quality of IR $\mu$ PIV recordings, allowing IR $\mu$ PIV to be used in lower signal-to-noise ratio environments. An expression for the depth of field of a PIV system using a CCD was presented and a more general expression for the measurement depth based on Meinhart et al.'s [12] work was developed. These expressions are not only valid for IR $\mu$ PIV but for $\mu$ PIV in general.

For lower flow rates $(\operatorname{Re}=10.2)$, little flow maldistribution was observed (only $2.4 \%$ ). Higher flow rates $(\mathrm{Re}=102)$ show a significant flow maldistribution, where the maximum mass flux ratio 
is $29.9 \%$ greater than the minimum. For $\operatorname{Re}=102$, channels near the lateral edge have a substantially lower flow rate than channels near the centerline, which would have a detrimental effect on the heat transfer performance of the microchannel heat sink.

The fluid flow performance of the heat sink was further explored numerically. The numerical results were found to be in very good agreement with the experimental data. The numerical results indicate that changing the manifold shape to a rectangular design yields a significantly lower flow maldistribution (4.7\%) compared to the original manifold design (33.5\%). Furthermore, the pressure drop between the inlet and exit ports was $32.7 \%$ greater for the original manifold compared to the rectangular design, which further illustrates the importance of a properly designed manifold to maximize the performance of a microchannel heat sink.

\section{Acknowledgements}

Financial support from the Indiana 21st Century Research and Technology Fund is gratefully acknowledged. The authors also acknowledge Sandia National Laboratories for fabrication of the silicon microchannel heat sink and Dong Liu for assistance in various aspects of the work. Dr. Jayathi Murthy is thanked for her helpful discussions regarding the numerical work.

\section{References}

[1] D. B. Tuckerman, R. F.W. Pease, High-performance heat sinking for VLSI, IEEE Electron Device Letters 2 (1981) 126-129.

[2] D. Liu, S.V. Garimella, Investigation of liquid flow in microchannels, Journal of Thermophysics and Heat Transfer, 18 (1) (2004) 65-27.

[3] P.-S. Lee, S.V. Garimella, D. Liu, Heat transfer in rectangular microchannels, International Journal of Heat and Mass Transfer 48 (2005) 1688-1704.

[4] M.-C. Lu, B.-C. Yang, C.-C. Wang, Numerical study of flow mal-distribution on the flow and heat transfer for multi-channel cold-plates, Proceedings of the 20th IEEE SEMI-THERM Symposium, San Jose, CA, 2004, pp. 205-212.

[5] O. Tonomura, S. Tanaka, M. Noda, M. Kano, S. Hasebe, I. Hashimoto, CFD-based optimal design of manifold in plate-fin microdevices, Chemical Engineering Journal 101 (2004) 397402. 
[6] J.G. Santiago, S.T. Wereley, C.D.,Meinhart, D.J. Beebe, R.J. Adrian, A particle image velocimetry system for microfluidics, Experiments in Fluids 25 (1998) 316-319.

[7] C.D. Meinhart, S.T. Wereley, J.G. Santiago, PIV measurements of microchannel flow, Experiments in Fluids 27 (1999) 414-419.

[8] G. Han, J.C. Bird, K. Johan, A. Westin, Z. Cao, K. Breuer, Infrared diagnostics for measuring of fluid and solid motion inside silicon microdevices, Microscale Thermophysical Engineering 8 (2) (2004) 169-182.

[9] D. Liu, S.V. Garimella, S.T. Wereley, Infrared micro-particle image velocimetry in siliconbased microdevices, Experiments in Fluids 38 (2005) 385-392.

[10] E.D. Palik, Handbook of Optical Constants of Solids: Vol. 1, Academic Press, New York, 1985.

[11] J.S. Lim, Two-Dimensional Signal and Image Processing, Prentice Hall, Englewood Cliffs, NJ, 1990.

[12] C.D. Meinhart, S.T. Wereley, M.H.B. Gray, Volume illumination for two-dimensional particle image velocimetry, Measurement Science and Technology 11 (2000) 809-814.

[13] S. Inoué, R. Oldenbourg, Microscopes, in: M. Bass, ed., Handbook of Optics, Vol. 2, McGraw-Hill, New York, 1995, 17.1-17.52.

[14] A.K. Prasad, R.J. Adrian, C.C. Landreth, P.W. Offut, Effect of resolution on the speed and accuracy of particle image velocimetry interrogation, Experiments in Fluids 13 (1993) 105116.

[15] C.D. Meinhart, S.T. Wereley, The theory of diffraction limited resolution in microparticle image velocimetry, Measurement Science and Technology 14 (2003) 1047-1053.

[16] P.-S. Lee, S.V. Garimella, Thermally developing flow and heat transfer in rectangular microchannels of different aspect ratios, International Journal of Heat and Mass Transfer 49 (2006) 3060-3067.

[17] FLUENT 6 User's Guide, Fluent Inc, Lebanon, NH, 2000.

[18] S.T. Wereley, C.D. Meinhart, Second-order accurate particle image velocimetry, Experiments in Fluids 31 (2001) 258-268.

[19] L. Gui, S.T. Wereley, A correlation-based continuous window-shift technique to reduce the peak-locking effect in digital PIV image evaluation, Experiments in Fluids 32 (2002) 506517. 
[20] C.D. Meinhart, S.T. Wereley, J.G. Santiago, A PIV algorithm for estimating time-averaged velocity fields, Journal of Fluids Engineering 122 (2000) 285-289.

[21] G.B. Whitham, The Navier-Stokes equations of motion, in: Rosenhead, L., ed., Laminar Boundary Layers, Oxford University Press, Oxford, 1963, pp. 114-162. 


\section{List of Figures}

Figure 1. IR $\mu$ PIV system configuration.

Figure 2. Microchannel test piece. An image of the test piece, with channels fabricated in a silicon substrate, a glass cover plate, and stainless steel tubing ports, is shown in (a). The dimensions of the silicon substrate (in $\mathrm{mm}$ ) are shown in (b).

Figure 3. Variation in channel depth at mid-length of heat $\operatorname{sink}(x=4.5 \mathrm{~mm})$.

Figure 4. Flow loop for higher flow rates $(\operatorname{Re}=102)$.

Figure 5. Image-processing operations developed to enhance the quality of the PIV recording: (a) original image recording, (b) background image, (c) image after background subtraction, (d) image after unsharp mask, (e) image after thresholding, (f) image after local contrast enhancement, and (g) image after unpaired particle removal. Inset in (a) shows enlarged view of a double-exposed particle pair. The contrast has been enhanced in the inset to highlight the particles.

Figure 6. Computational domain for the simplified numerical model. The geometry of the domain is shown in (a). The manifolds have uniform depth of $421 \mu \mathrm{m}$ while the channels are $371 \mu \mathrm{m}$ deep. The inlet and outlet is modeled using pressure boundary conditions applied to cylindrical surfaces cut into the manifold regions. See Figure $2 b$ for the remaining dimensions of the heat sink. An enlarged representation of the meshing at the junction of the manifold and channels is shown in (b).

Figure 7. Velocity profile in channel 35 at $\mathrm{Re}=102$.

Figure 8. Flow distribution in the heat sink: (a) velocity profiles at $\operatorname{Re}=10.2$, (b) velocity profiles at $\operatorname{Re}=102$, and (c) mass flux distribution.

Figure 9. Comparison of numerical and experimental results for the mass flux distribution.

Figure 10. Predictions of flow distribution using a rectangular manifold design. The dimensions of the computational domain are shown in (a). The depth of the manifolds and channels is the same as the original manifold design. The comparison of mass flux distribution at $\mathrm{Re}=102$ between the original and rectangular manifold designs is shown in (b).

Figure 11. Streamlines and velocity magnitude contours shown at mid-height with respect to the channels in (a) existing manifold design and (b) rectangular manifold design. 


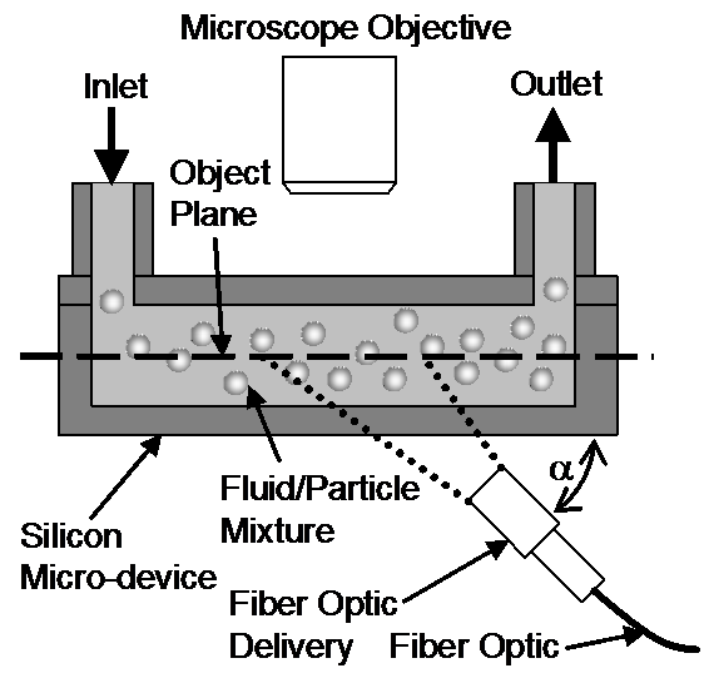

Figure 1 . IR $\mu$ PIV system configuration. 


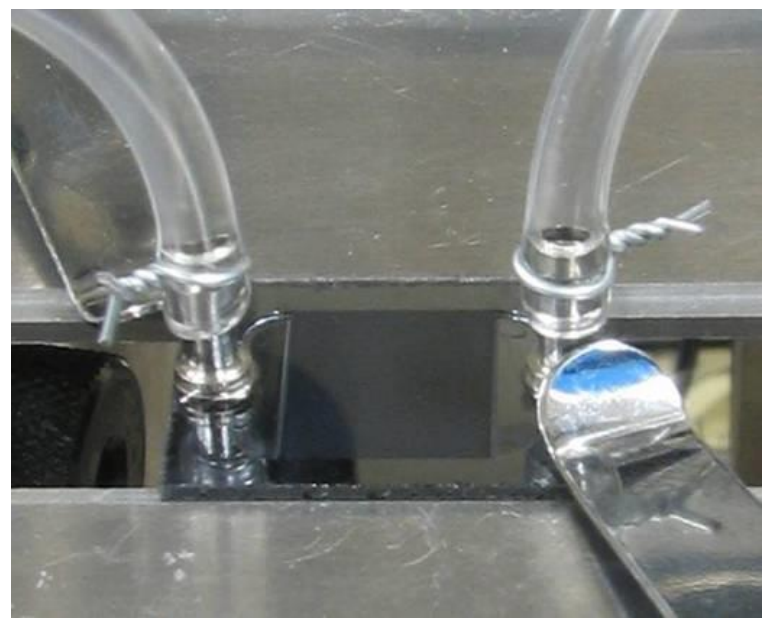

(a)

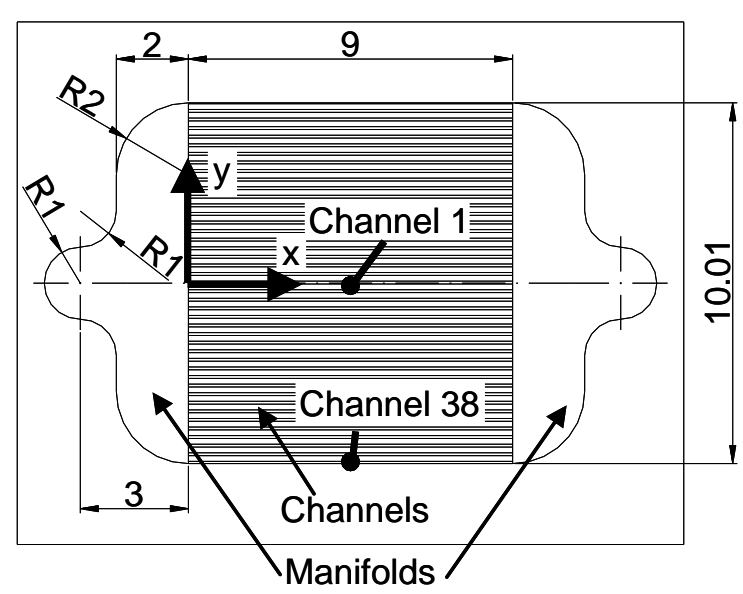

(b)

Figure 2. Microchannel test piece. An image of the test piece, with channels fabricated in a silicon substrate, a glass cover plate, and stainless steel tubing ports, is shown in (a). The dimensions of the silicon substrate (in $\mathrm{mm}$ ) are shown in (b). 


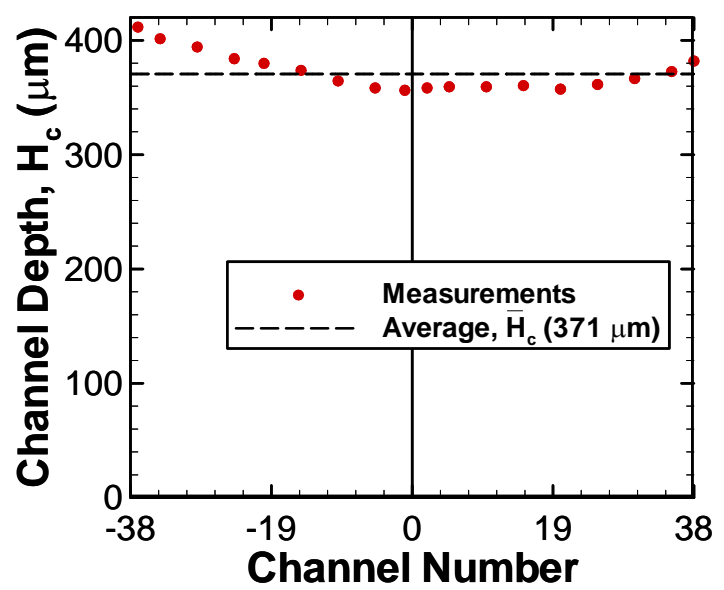

Figure 3. Variation in channel depth at mid-length of heat $\operatorname{sink}(x=4.5 \mathrm{~mm})$. 


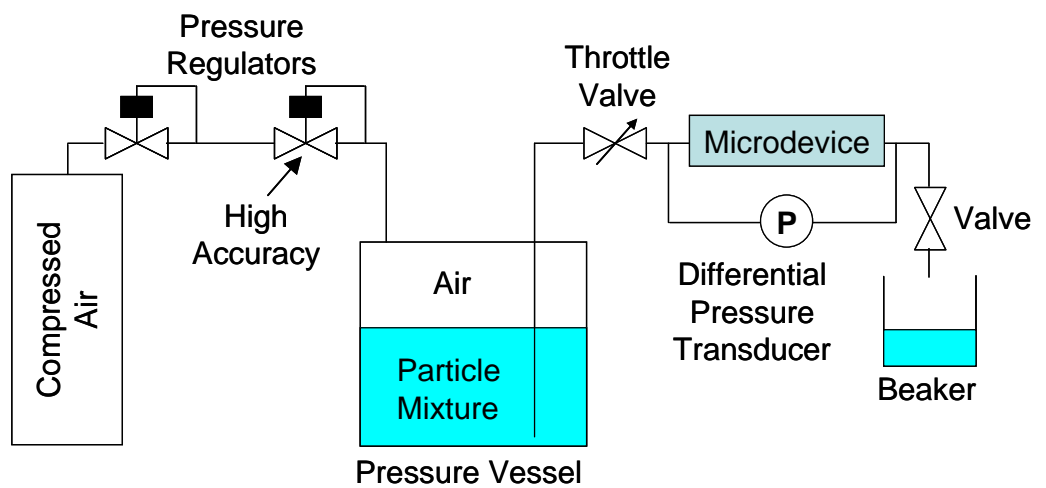

Figure 4. Flow loop for higher flow rates $(\operatorname{Re}=102)$. 


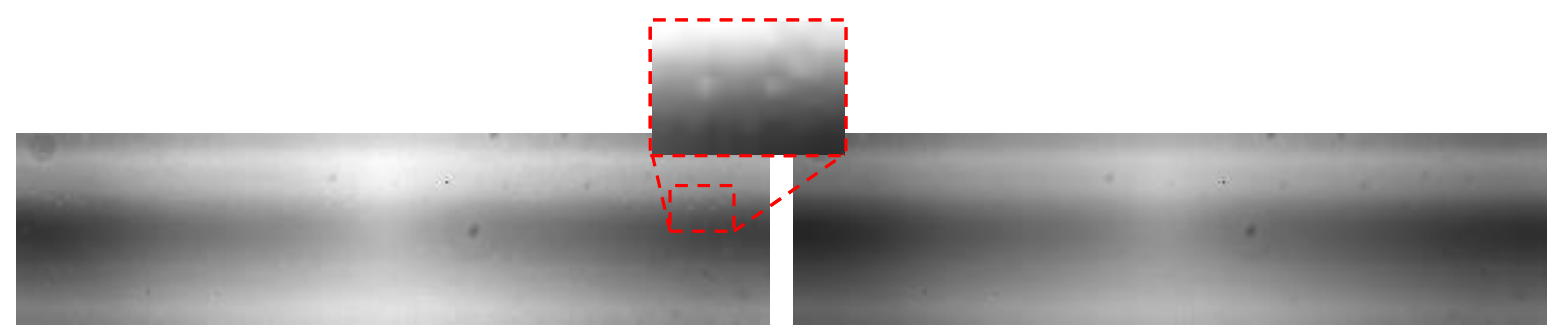

(a)

(b)

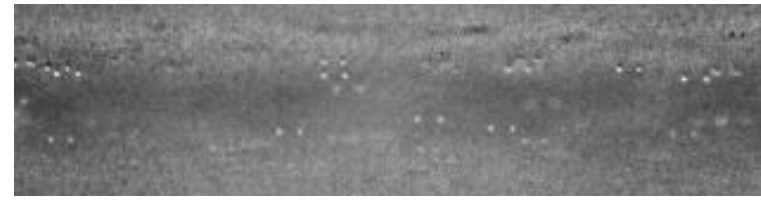

(c)

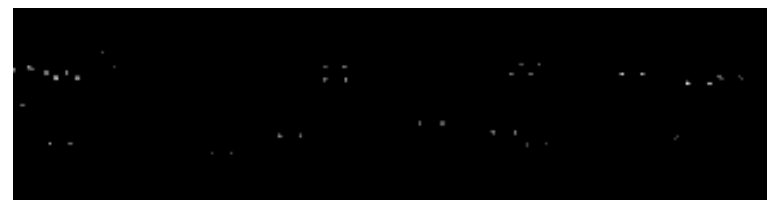

(e)

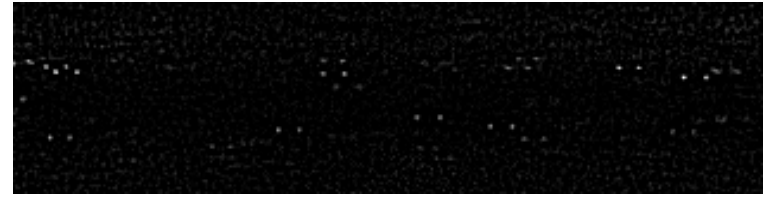

(d)

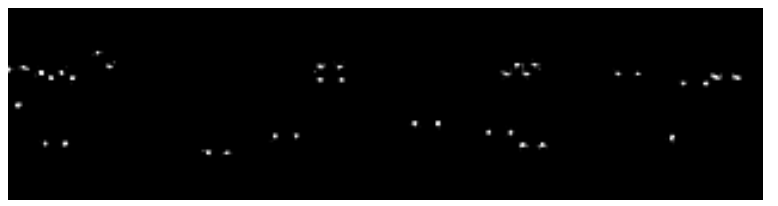

(f)

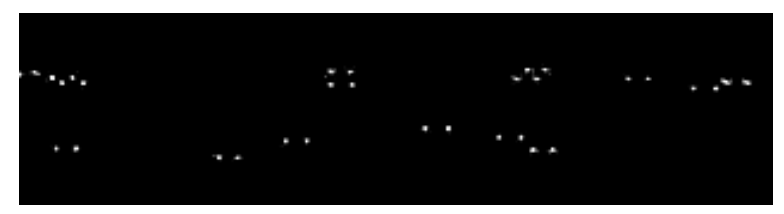

(g)

Figure 5. Image-processing operations developed to enhance the quality of the PIV recording: (a) original image recording, (b) background image, (c) image after background subtraction, (d) image after unsharp mask, (e) image after thresholding, (f) image after local contrast enhancement, and (g) image after unpaired particle removal. Inset in (a) shows enlarged view of a double-exposed particle pair. The contrast has been enhanced in the inset to highlight the particles. 


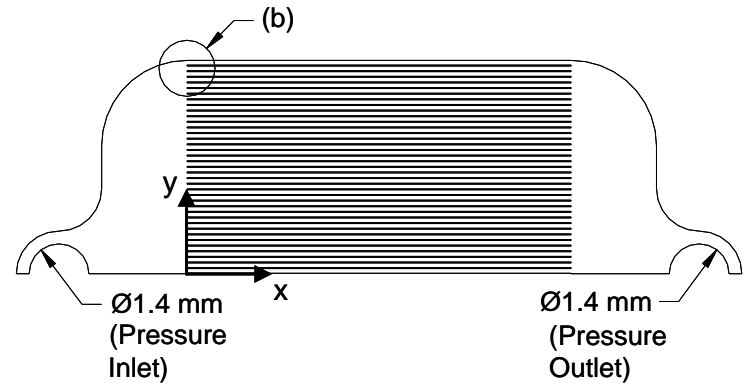

(a)

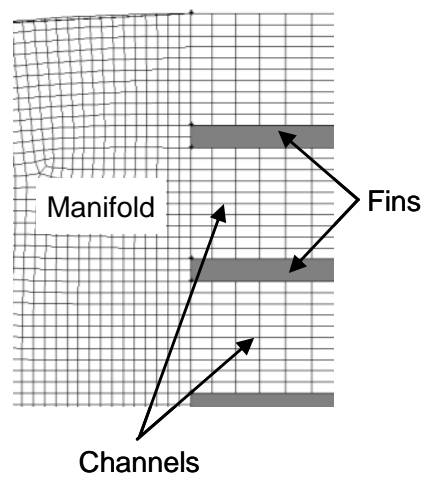

(b)

Figure 6. Computational domain for the simplified numerical model. The geometry of the domain is shown in (a). The manifolds have uniform depth of $421 \mu \mathrm{m}$ while the channels are $371 \mu \mathrm{m}$ deep. The inlet and outlet is modeled using pressure boundary conditions applied to cylindrical surfaces cut into the manifold regions. See Figure $2 b$ for the remaining dimensions of the heat sink. An enlarged representation of the meshing at the junction of the manifold and channels is shown in (b). 


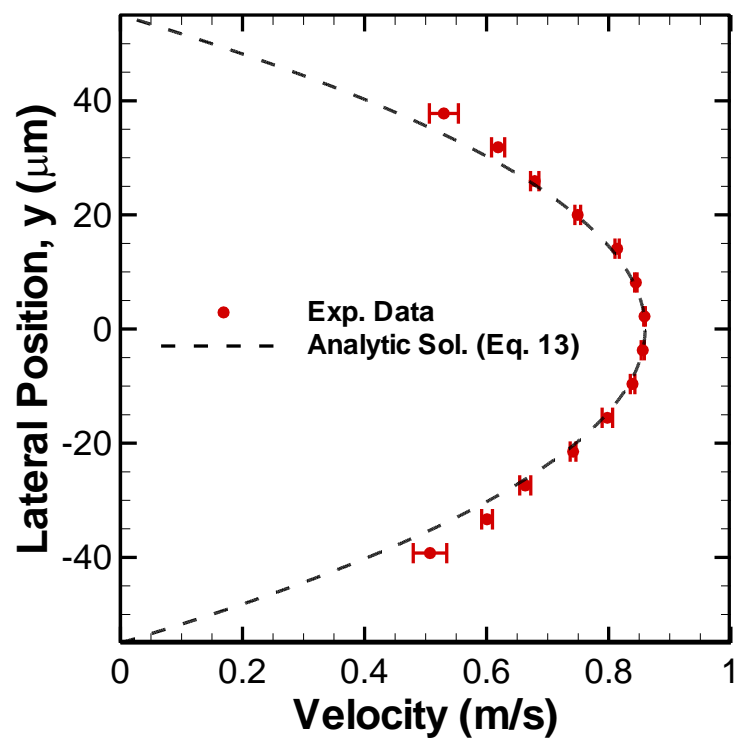

Figure 7. Velocity profile in channel 35 at $\operatorname{Re}=102$. 


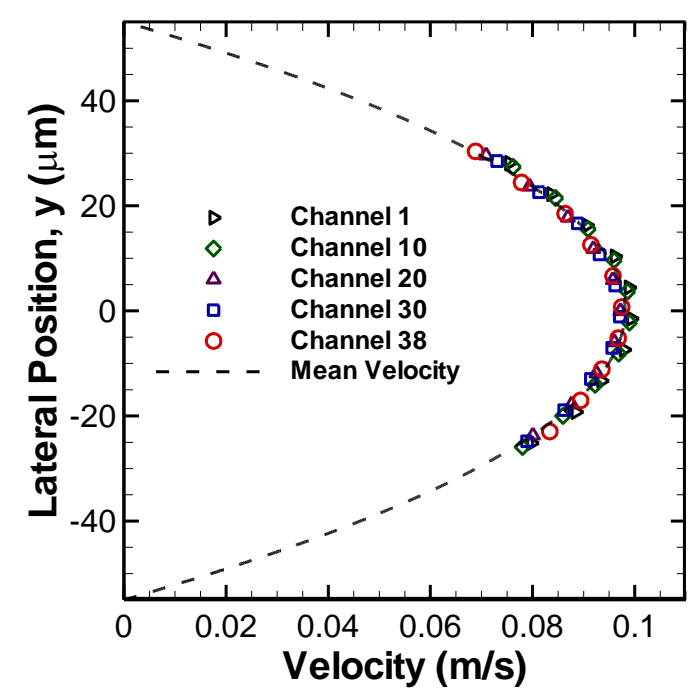

(a)

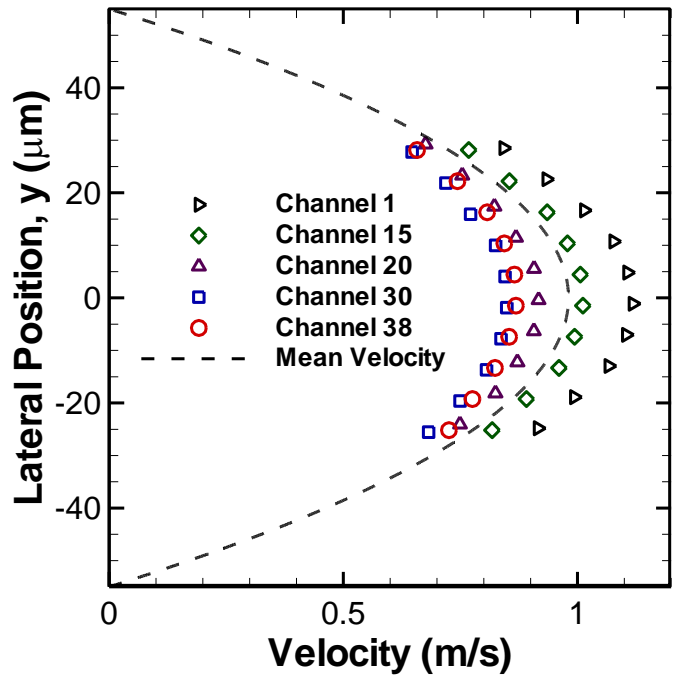

(b)

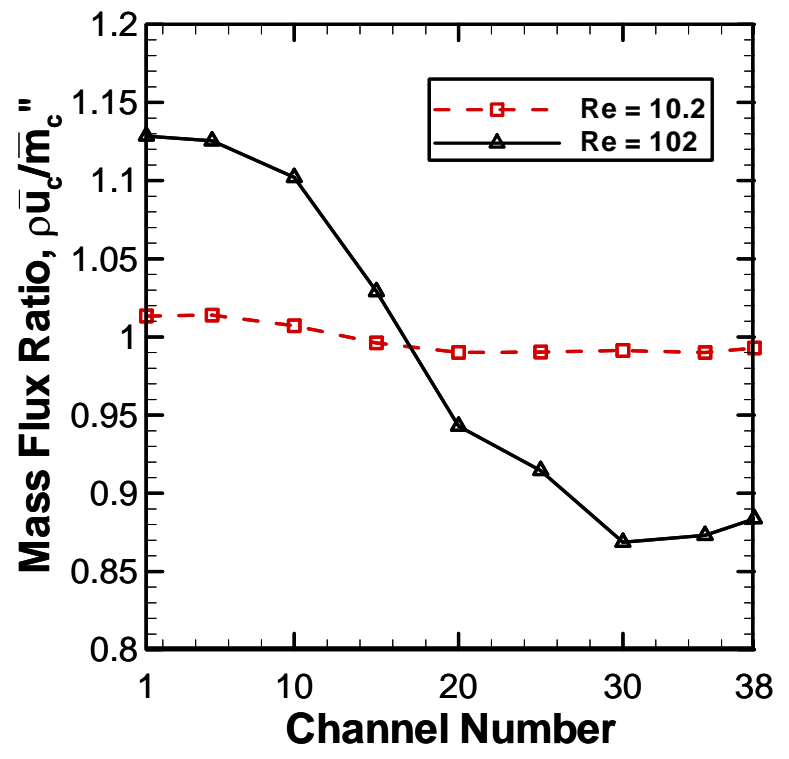

(c)

Figure 8. Flow distribution in the heat sink: (a) velocity profiles at $\operatorname{Re}=10.2$, (b) velocity profiles at $\operatorname{Re}=102$, and (c) mass flux distribution. 


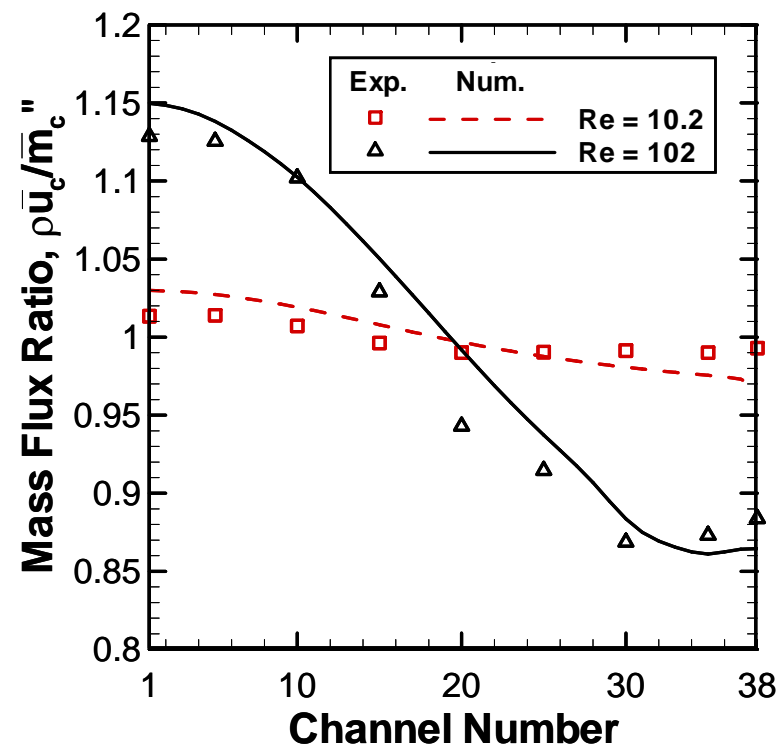

Figure 9. Comparison of numerical and experimental results for the mass flux distribution. 


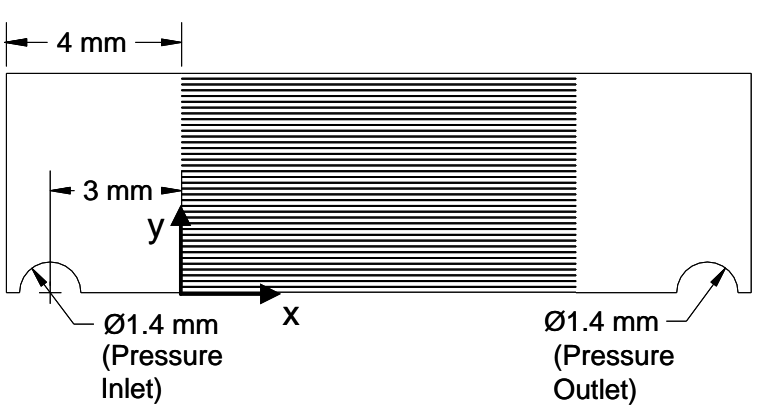

(a)

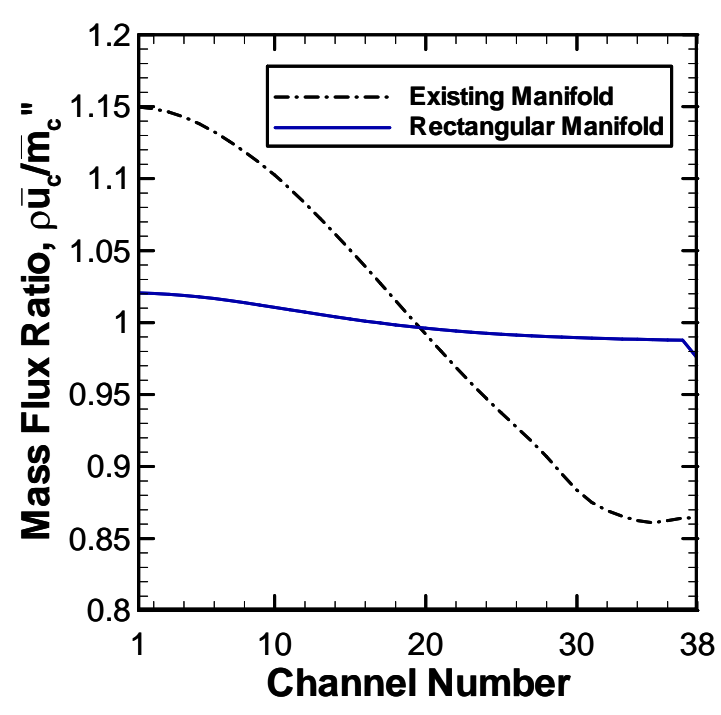

(b)

Figure 10. Predictions of flow distribution using a rectangular manifold design. The dimensions of the computational domain are shown in (a). The depth of the manifolds and channels is the same as the original manifold design. The comparison of mass flux distribution at $\mathrm{Re}=102$ between the original and rectangular manifold designs is shown in (b). 


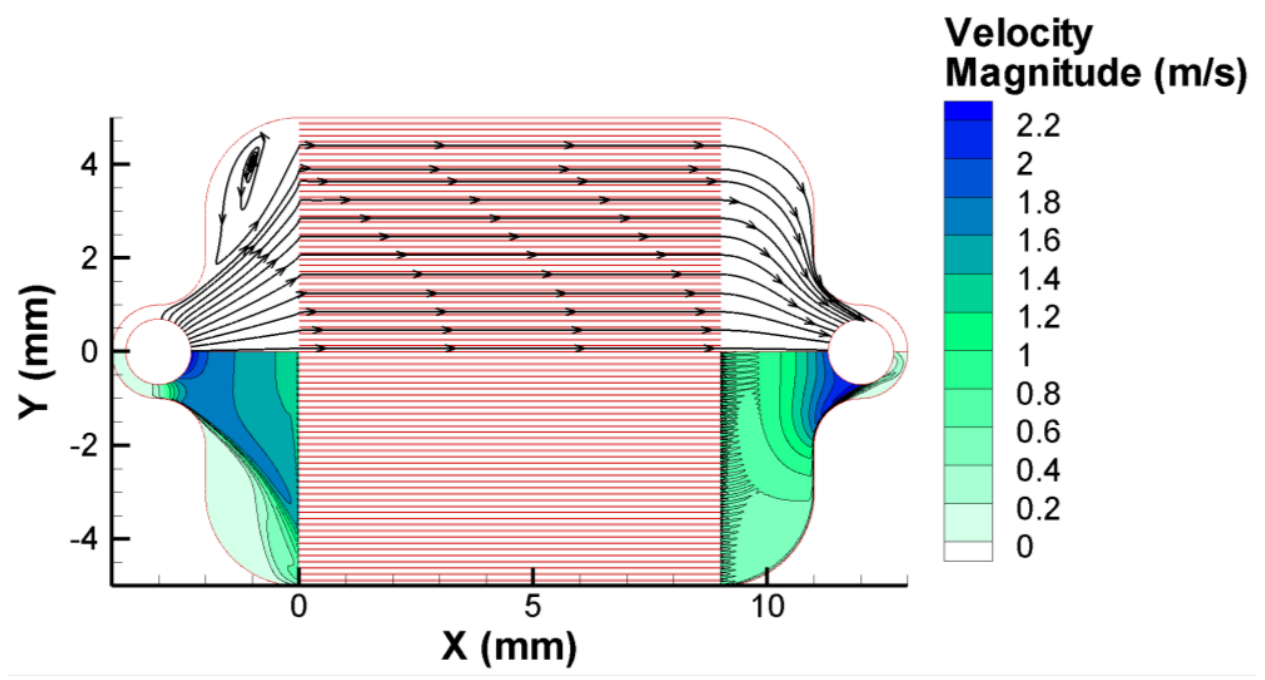

(a)

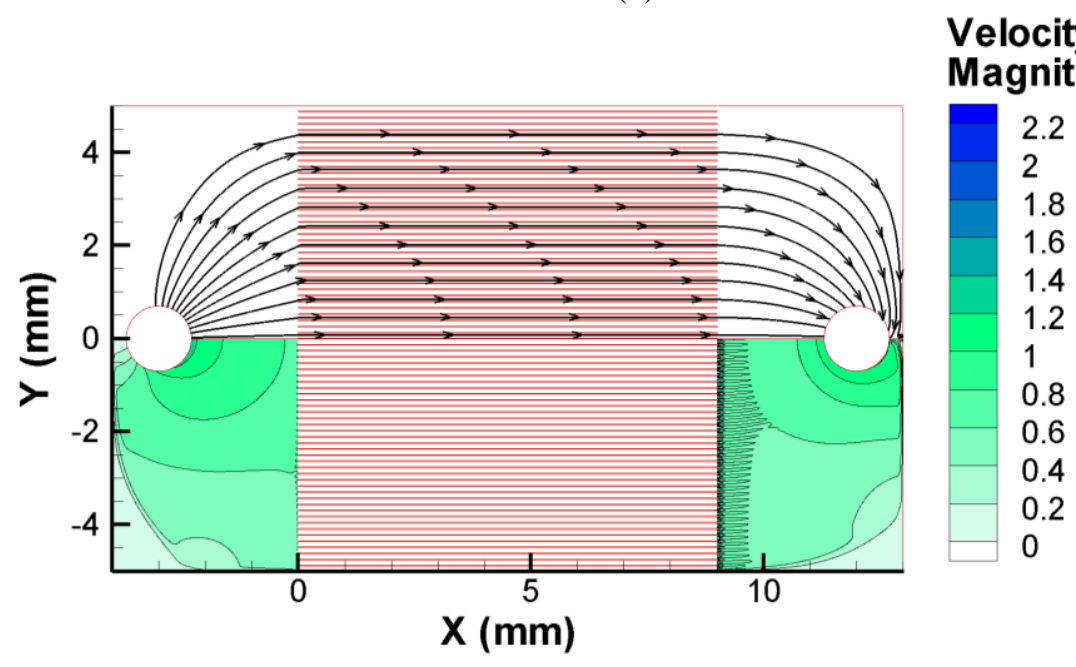

(b)

Figure 11. Streamlines and velocity magnitude contours shown at mid-height with respect to the channels in (a) existing manifold design and (b) rectangular manifold design. 